\title{
Compost de guano de gallina en la composición de sustratos para la producción de plantines florales
}

\author{
Barbaro, L.A.; M.A. Karlanian, P.F. Rizzo, N.I. Riera, V. Della Torre, M. Beltrán y D.E. Crespo
}

\begin{abstract}
RESUMEN
Se evaluaron sustratos formulados con distintas proporciones (20, 50 y $80 \%$ ) de tres tipos de compost de guano de gallina $\left(\mathrm{CGG}_{1}, \mathrm{CGG}_{2}, \mathrm{CGG}_{3}\right)$ mezclados con compost de corteza de pino y un sustrato comercial. Los diez tratamientos fueron usados para el cultivo de plantines de Impatiens walleriana y Salvia splendens. Se caracterizó física y químicamente cada sustrato, y sobre los plantines se evaluó sobrevivencia, masa seca y calcio, magnesio, potasio y sodio en la hoja. Los sustratos con $20 \%$ y $50 \%$ de los tres CGG tuvieron la mayor capacidad de retención de agua, y con $80 \%$, la mayor porosidad de aire. Los sustratos con $50 \%$ y $80 \%$ presentaron los más altos valores de $\mathrm{pH}(\geq 6,6)$, potasio y sodio. Para las mismas proporciones, $\mathrm{CGG}_{2}$ y $\mathrm{CGG}_{3}$ presentaron valores de $C E>1,1 \mathrm{dS} \mathrm{cm}^{-1}$. La mayor masa seca de los plantines se logró para el sustrato comercial, los sustratos con 20\% de los tres CGG y con 50\% de $C_{G} G_{1}$. Estos sustratos exhibieron en las hojas una mayor concentración de calcio y magnesio, y menor de potasio. Las mejores características de sustratos para el desarrollo de los plantines evaluados se lograron en los sustratos con $20 \%$ de los tres CGG y con $50 \%$ del compost $C_{\text {. }}$.
\end{abstract}

Palabras clave: sustrato, compost de guano de gallina, plantines florales.

Barbaro, L.A.; M.A. Karlanian, P.F. Rizzo, N.I. Riera, V. Della Torre, M. Beltrán and D.E. Crespo, 2013. Poultry compost in the composition of substrates for flower seedlings production. Agriscientia 30 (1): 25-35

\section{SUMMARY}

Substrates formulated with different proportions (20,50 and $80 \%$ ) of three types of poultry compost $\left(\mathrm{CGG}_{1}, \mathrm{CGG}_{2}, \mathrm{CG}_{3}\right)$ mixed with pine bark compost and commercial substrate were evaluated. The ten treatments were used to cultivate seedlings of walleriana Impatiens and Salvia splendens. Each substrate was characterised physically and chemically, and the survival, dry mass and calcium, magnesium, potassium and sodium in the leaf were evaluated in seedlings. Substrates with 20\% and 50\% among the three CGG presented the highest water retention capacity and, with $80 \%$ the highest air porosity. The substrates with $50 \%$ and $80 \%$ showed the highest values of $\mathrm{pH}(\geq 6.6)$, potassium and sodium. For the same proportions, $\mathrm{CGG}_{2}$ and $\mathrm{CGG}_{3}$ presented 
CE values $>1.1 \mathrm{dS} \mathrm{cm}^{-1}$. The highest dry weight of the seedlings was achieved with the commercial substrate, the substrates with 20\% of the three CGG and with $50 \%$ of $\mathrm{CGG}_{1}$. These substrates showed a higher concentration of calcium and magnesium, and a lower concentration of potassium in the leaves. The best characteristics of substrates for the development of the seedlings tested were achieved in the substrates with $20 \%$ of the three CGG and with $50 \%$ of compost $\mathrm{CGG}_{1}$.

Key words: substrate, poultry compost, flower seedlings.

Barbaro L.A. y M.A. Karlanian: Instituto de Floricultura del INTA, De los Reseros y Las Cabañas, 1686 Hurlingham, Buenos Aires, Argentina. P.F. Rizzo, N.I. Riera, V. Della Torre y D.E. Crespo: Instituto de Microbiología y Zoología Agrícola del INTA, De los Reseros y Las Cabañas, 1686 Hurlingham, Buenos Aires, Argentina. M. Beltrán: Instituto de Suelos del INTA, De los Reseros y Las Cabañas, 1686 Hurlingham, Buenos Aires, Argentina. Correspondencia a L. A. Barbaro: lbarbaro@cnia.inta.gov.ar

\section{INRODUCCIÓN}

A través de los años se vienen evaluando diferentes materiales y mezclas, para lograr desarrollar un sustrato que posea las características apropiadas para el cultivo de plantas florales en macetas. En el mundo, el sustrato más común para tal fin es preparado con turba de Sphagnum, debido a sus mejores propiedades físicas y químicas, así como también por su baja velocidad de degradación (Garcia-Gomez et al., 2002). En la Argentina se continúa utilizando suelo, pero debido a las malas condiciones físicas y a la proliferación de patógenos no es recomendable utilizar este material en la formulación de sustratos (Ansorena Miner, 1994). Las desventajas del suelo mineral y el costo de la turba de calidad, junto con la menor disponibilidad de ambos materiales que se prevé en un futuro próximo debido a las limitaciones ambientales, hacen que sea necesario buscar materiales alternativos (Raviv et al, 1986; Abad et al, 2001).

Una de las alternativas actualmente estudiada en el mundo es el compost. Varios residuos y subproductos han sido utilizados como material para producir compost y evaluados como componentes de sustratos para la producción de plantas (Estévez-Schwarz et al., 2009). Es así que los materiales compostados se han usado exitosamente para un amplio espectro de especies, desde plantines hortícolas y florales (Grigatti et al., 2007; Hong et al., 2010) hasta forestales (Landis \& Morgan, 2009).

Uno de los compost que podría ser evaluado para su uso como componente de sustrato es el compost de guano de gallina. Hoy la avicultura mundial se encuentra en constante crecimiento y la Argentina mantiene esta tendencia, debida al mayor grado de intensificación que han tenido estos sistemas productivos en los últimos años. Este hecho ha generado una gran acumulación de residuos orgánicos en áreas pequeñas, y el compostaje es un proceso que permitiría estabilizar este tipo de residuos para su reutilización en la agricultura (Ministerio de Agricultura, Ganadería y Pesca de la Nación Argentina, 2010).

El compost de guano de gallina, en general, presenta $\mathrm{pH}$ alcalino y conductividad eléctrica elevada (Burés, 1997), y por tal motivo es conveniente mezclarlo con otros materiales de menor $\mathrm{pH}$ y niveles de sales. Entre estos materiales se encuentra el compost de corteza de pino, el cual es el producto final del compostado de cortezas provenientes de aserraderos y descortezadoras de madera (Burés, 1997). Generalmente tiene pH entre 4 y 7 , baja conductividad eléctrica $\left(0,1-0,6 \mathrm{dS} \mathrm{cm}^{-1}\right)$ y sus propiedades físicas, como porosidad total, retención de agua, porosidad de aireación entre otras, varían según el tamaño de partícula (Burés, 1997; Villa Castillo, 2004). También es un material empleado como supresor de algunos patógenos, entre ellos, Phytophthora y Rhizoctonia (Nelson \& Hoitink, 1982; Spencer \& Benson, 1982).

Para obtener un sustrato de calidad se deben tener en cuenta una serie de propiedades que dependerán del sistema del cultivo, especie y condiciones climáticas. En este sentido, las principales propiedades físicas que requiere un buen sustrato 
son densidad adecuada y buena distribución de las partículas para obtener una óptima distribución del tamaño de los poros y, como consecuencia, el porcentaje que contendrá de aire y agua (Fonteno, 1999; Raviv \& Lieth, 2008). Una correcta aireación aportará oxígeno al sistema radicular y permitirá la evacuación del gas carbónico producido por las raíces y microorganismos (Lemaire et al., 2005). Por otro lado, una adecuada proporción de poros con capacidad de retención hídrica permitirá a la planta obtener el agua y los nutrientes necesarios para su desarrollo. Entre las principales propiedades químicas responsables de la calidad del sustrato se mencionan al pH y la conductividad eléctrica (Handreck $\&$ Black, 2002). Es recomendable que el sustrato presente un $\mathrm{pH}$ entre 5,5 y 6,8, para que los nutrientes se encuentren disponibles, y una baja conductividad eléctrica, para que no existan problemas de toxicidad por sales (Landis et al., 2000).

Una vez realizada la caracterización física y química del sustrato, para considerar que se encuentra en condiciones de ser utilizado, se deberá pasar a una próxima etapa, en donde se evaluará su capacidad de producir plantas en condiciones óptimas. Para esto se recomienda realizar ensayos de crecimiento vegetal con diversas especies (Abad et al., 1993).

El objetivo de este trabajo fue evaluar el uso de diferentes sustratos formulados con distintas proporciones de compost de guano de gallina y compost de corteza de pino mediante el desarrollo de plantines de coral (Salvia splendens L.) y alegría del hogar (Impatiens walleriana hybrids Hook. f.).

\section{MATERIALES Y MÉTODOS}

\section{Compost, sustratos formulados y sustrato comercial}

Los compost de guano de gallina evaluados fueron elaborados en el Instituto de Microbiología y Zoología Agrícola (IMYZA) del INTA durante un período de 83 días. El residuo avícola a procesar fue obtenido de gallinas ponedoras en producción criadas en galpones automatizados con instalaciones tipo Zucami, situados en una granja en la localidad de Mercedes (Buenos Aires, Argentina).

La mezcla inicial de cada compost de guano de gallina (CGG) evaluado estaba compuesta por los siguientes materiales:

Compost de guano de gallina $1\left(\mathrm{CGG}_{1}\right): 40 \%$ guano de gallinas ponedoras, 20\% marlo de maíz triturado, 20\% de aserrín y $20 \%$ viruta.

Compost de guano de gallina $2\left(\mathrm{CGG}_{2}\right): 60 \%$ guano de gallinas ponedoras, 20\% marlo de maíz triturado y $20 \%$ de aserrín.

Compost de guano de gallina $3\left(\mathrm{CGG}_{3}\right): 60 \%$ de guano de gallinas, 30\% de marlo de maíz entero y 10\% de marlo de maíz triturado.

Cada mezcla inicial fue homogeneizada con un mezclador con capacidad para 0,50 $\mathrm{m}^{3}$, para luego armar una pila de $2 \mathrm{~m}^{3}$ con $1 \mathrm{~m}$ de altura. Las pilas fueron aireadas manualmente cada 3 días durante la fase mesófila y termófila, y cada 5 días durante la fase de enfriamiento y maduración. El contenido de humedad se mantuvo en un $60 \%$ mediante riego manual. Semanalmente, durante el proceso de compostaje en tres puntos equidistantes se midió la temperatura de cada pila (Figura 1).

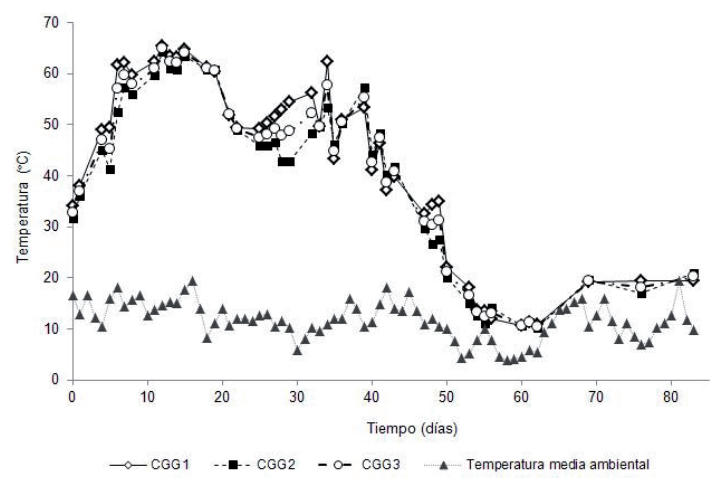

Figura 1. Temperaturas registradas durante el proceso de compostaje de los tres compost de guano de gallina (CGG).

En la Tabla 1 se presentan las propiedades físicas y químicas analizadas a los compost utilizados. Según los límites establecidos por TMECC (2001) e Iglesias Jiménez et al. (2008), cuyos valores de relación carbono/nitrógeno $(\mathrm{C} / \mathrm{N})$ deben ser menor a 20, el índice respirométrico estático $\left(\mathrm{IRE}_{\mathrm{MO}}\right)$ menor a $0,5 \mathrm{mg} \mathrm{O} 2 \mathrm{~g}^{-1} \mathrm{MO} \mathrm{h} \mathrm{h}^{-1}$ y la relación amonio/nitrato $\left(\mathrm{N}-\mathrm{NH}_{4+} / \mathrm{N}_{-} \mathrm{NO}_{3}\right)$ menor a 0,16 , los tres compost de guano de gallina se encontraban estables y maduros.

Cada compost de guano de gallina fue mezclado en 20,50 y $80 \%$ con compost de corteza de pino (CP). Los nueve sustratos formulados fueron evaluados junto a un sustrato comercial utilizado como testigo, formulado con turba Sphagnum como principal componente, además de compost de corteza de pino, vermiculita y perlita (Tabla 1).

Cada sustrato conformó un tratamiento: 1) $80 \%$ $\mathrm{CGG}_{1}+20 \% \mathrm{CP}$; 2) $50 \% \mathrm{CGG}_{1}+50 \% \mathrm{CP}$; 3) $20 \%$ $\left.\left.\mathrm{CGG}_{1}+80 \% \mathrm{CP} ; 4\right) 80 \% \mathrm{CGG}_{2}+20 \% \mathrm{CP} ; 5\right) 50 \%$ $\left.\left.\mathrm{CGG}_{2}+50 \% \mathrm{CP} ; 6\right) 20 \% \mathrm{CGG}_{2}+80 \% \mathrm{CP} ; 7\right) 80 \%$ $\mathrm{CGG}_{3}+20 \% \mathrm{CP}$; 8) $50 \% \mathrm{CGG}_{3}+50 \% \mathrm{CP}$; 9) $20 \%$ $\left.\mathrm{CGG}_{3}+80 \% \mathrm{CP} ; 10\right)$ sustrato comercial. 


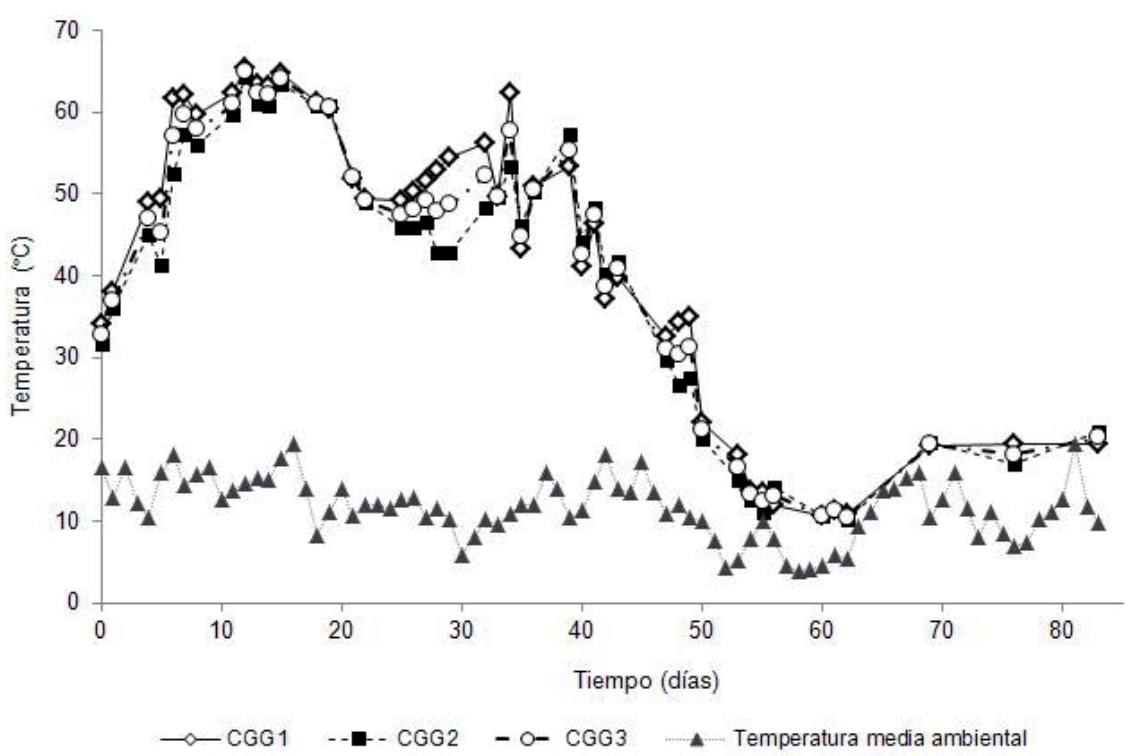

Figura 1. Temperaturas registradas durante el proceso de compostaje de los tres compost de guano de gallina (CGG).

Tabla 1. Relación carbono/nitrógeno $(\mathrm{C} / \mathrm{N})$, amonio/nitrato $\left(\mathrm{N}-\mathrm{NH}_{4+} / \mathrm{N}-\mathrm{NO}_{3}\right)$, índice respirométrico estático (IRE $\left.\mathrm{MO}_{\mathrm{MO}}\right)$, densidad aparente (Dap), espacio poroso total (EPT), capacidad de retención de agua (CRA), poros con aire (PA), materia orgánica (MO), pH, conductividad eléctrica $(\mathrm{CE})$, calcio $(\mathrm{Ca})$, magnesio $(\mathrm{Mg})$, potasio $(\mathrm{K})$ y sodio $(\mathrm{Na})$ de los compost de guano de gallina 1,2 y $3\left(\mathrm{CGG}_{1}, \mathrm{CGG}_{2}\right.$ y $\mathrm{CGG}_{3}$ ), del compost de corteza de pino (CP) y del sustrato comercial.

\begin{tabular}{|c|c|c|c|c|c|c|}
\hline Variables & Unidades & $\mathrm{CGG}_{1}$ & $\mathrm{CGG}_{2}$ & $\mathrm{CGG}_{3}$ & $\mathrm{CP}$ & $\begin{array}{l}\text { Sustrato } \\
\text { comercial }\end{array}$ \\
\hline $\mathrm{C} / \mathrm{N}$ & & 14,4 & 13,6 & 18,1 & & \\
\hline $\mathrm{N}-\mathrm{NH}_{4} / \mathrm{N}_{-} \mathrm{NO}_{3}$ & $\mathrm{mg} \mathrm{g}^{-1}$ & 0,002 & 0,006 & 0,004 & & \\
\hline $\mathrm{IRE}_{\mathrm{MO}}$ & $\mathrm{mg} \mathrm{O}_{2} \mathrm{~g}^{-1} \mathrm{MOh}^{-1}$ & 0,25 & 0,45 & 0,37 & & \\
\hline Dap & $\mathrm{kg} \mathrm{m}^{-3}$ & 400 & 500 & 500 & 180 & 450 \\
\hline EPT & $\%$ & 78 & 72 & 73 & 88 & 87 \\
\hline CRA & $\%$ & 42 & 46 & 46 & 33 & 51 \\
\hline PA & $\%$ & 36 & 26 & 27 & 46 & 36 \\
\hline $\mathrm{MO}$ & $\%$ & 84 & 87 & 87 & 75 & 56 \\
\hline $\mathrm{pH}$ & & 8,8 & 9,0 & 8,7 & 4,3 & 5,1 \\
\hline $\mathrm{CE}$ & $\mathrm{dS} \mathrm{m}^{-1}$ & 1,4 & 2,0 & 2,7 & 0,17 & 0,9 \\
\hline $\mathrm{Ca}$ & $\mathrm{g} \mathrm{L}^{-1}$ & 0,82 & 0,98 & 2,21 & 0,009 & 7,0 \\
\hline $\mathrm{Mg}$ & $g L^{-1}$ & 0,25 & 0,35 & 0,47 & 0,02 & 2,4 \\
\hline $\mathrm{K}$ & $g L^{-1}$ & 6,56 & 8,7 & 11,35 & 0,355 & 8,7 \\
\hline $\mathrm{Na}$ & $\mathrm{g} \mathrm{L}^{-1}$ & 2,01 & 3,39 & 2,88 & 0,16 & 5,0 \\
\hline
\end{tabular}

\section{Análisis de los sustratos, compost y agua para el riego}

Se elaboraron 200 litros de cada sustrato formulado y se guardó en una bolsa de polietileno negra. Se tomaron muestras de todos los sustratos abriendo sus respectivas bolsas y pasando su contenido a través de un tamiz con una malla de $20 \mathrm{~mm} \times 20$ $\mathrm{mm}$. Finalmente se mezcló todo el contenido de la bolsa para homogeneizar el lote. Se trazaron dos líneas oblicuas a cada lote tomando 10 litros de dos cuartos opuestos, conformando una muestra de 20 litros.

A cada muestra se le ajustó el porcentaje de humedad a un $50 \%( \pm 2)$ y se analizaron los siguientes parámetros por triplicado en el laboratorio de sustratos y aguas del Instituto de Floricultura:

Densidad aparente, con el método Hofmann (Fermino, 2003); espacio poroso total, capacidad de retención de agua y poros con aire con el método de De Boodt mediante los lechos de arena (De Boodt et al., 1974); pH y conductividad eléctrica (CE) en una relación 1+5 vol/vol de compost/agua (Barbaro et al., 2011). La concentración de calcio, magnesio, potasio y sodio en $\mathrm{g} \mathrm{L}^{-1}$ de sustrato, fueron analizados en el filtrado de la solución $1+5 \mathrm{vol} /$ vol con un espectrofotómetro de absorción atómica (Varian modelo 220 A) y los nitratos con electrodo ión selectivo (Orion modelo 920 A).

A los tres compost de guano de gallina, además de los análisis mencionados, se les analizó: relación carbono/nitrógeno $(\mathrm{C} / \mathrm{N})$ con los métodos de 
Walkey y Black para carbono y Kjeldhal para nitrógeno; amonio/nitrato $\left(\mathrm{N}-\mathrm{NH}_{4+} / \mathrm{N}_{-} \mathrm{NO}_{3}\right)$ mediante el método de micro destilación (Bremner, 1965) y el índice respirométrico estático basado en el contenido de materia orgánica (IRE ${ }_{\mathrm{MO}}$ ) (Barrena Gómez et al., 2006).

Para analizar el agua para el riego se tomó una muestra de medio litro en un recipiente limpio y enjuagado tres veces con el agua a analizar, luego de dejar correr el agua de la canilla cinco minutos. El análisis se realizó directamente sobre la muestra, y los parámetros fueron: $\mathrm{pH}$ y CE con un multiparamétrico (Horiba modelo F-54 BW); calcio, magnesio, potasio y sodio con un espectrofotómetro de absorción atómica (Varian modelo 220 A); nitratos con electrodo ión selectivo (Orion modelo 920 A); cloruros y bicarbonatos por titulación. El análisis se realizó en el laboratorio de sustratos y agua para riego del Instituto de Floricultura.

\section{Ensayos con plantas florales}

Los ensayos se realizaron en un invernáculo del Instituto de Floricultura del INTA (Hurlingham, Buenos Aires, República Argentina).

Cada ensayo estaba conformado por 10 tratamientos (nueve sustratos formulados con compost de guano de gallina y un sustrato comercial) con cinco repeticiones.

Se utilizaron: a) plantines de coral (Salvia splendens L.) var. Rojo y b) alegría de hogar (Impatiens walleriana hybrids Hook. f.) var. Accent Pink Imp. Ambas especies se trasplantaron el 17/11/11 en macetas de $13 \mathrm{~cm}$ de diámetro con capacidad para $900 \mathrm{~mL}$ de sustrato. El riego se realizó según demanda con agua de pozo, la misma que utilizan los productores de la zona, con un $\mathrm{pH}$ de 7,18; CE, 0,79 dS m ${ }^{-1}$; nitratos, 7,7 $\mathrm{mg} \mathrm{L}^{-1}$; calcio, 10,3 $\mathrm{mg} \mathrm{L}^{-1}$; magnesio, 7,5 $\mathrm{mg} \mathrm{L}^{-1}$; potasio, 12,1 $\mathrm{mg} \mathrm{L}^{-1}$; sodio, 144,6 mg L-1; cloruros, 24,3 $\mathrm{mg} \mathrm{L}^{-1}$ y bicarbonatos, $427 \mathrm{mg} \mathrm{L}^{-1}$.

Las temperaturas mínimas y máximas promedio registradas en el invernáculo durante ambos ensayos fueron de $15,2^{\circ} \mathrm{C}$ y $35,9^{\circ} \mathrm{C}$ respectivamente.

En los dos ensayos se fertilizó durante todo el ciclo sólo al tratamiento con sustrato comercial con un fertilizante compuesto 18-18-18 (NPK) con microelementos quelatados $(0,02 \% \mathrm{Cu}, 0,05 \% \mathrm{fe}$, $0,05 \% \mathrm{Mn}, 0.001 \% \mathrm{Mo}, 0,02 \% \mathrm{Zn})$. Las dosis fueron de $100 \mathrm{mg} \mathrm{L}^{-1}$ de N-P-K en la primera semana, $150 \mathrm{mg} \mathrm{L}^{-1}$ de N-P-K en la segunda, $200 \mathrm{mg} \mathrm{L}^{-1}$ de N-P-K en la tercera y finalmente, dos veces por semana con $200 \mathrm{mg} \mathrm{L}^{-1}$ de N-P-K en las restantes semanas. En estas últimas semanas también se fertilizó con la misma dosis a los tratamientos con sustratos formulados con $20 \%$ de compost de guano de gallina.

El ensayo con plantines de coral finalizó a los 34 días y con plantines de alegría del hogar a los 28 días desde el trasplante. En ese momento, a cinco plantines por tratamiento se les midió la materia seca de la parte aérea y radicular (secado en estufa a $60{ }^{\circ} \mathrm{C}$ hasta peso constante) expresado en unidades de masa. Además, se realizó un análisis químico a la materia seca aérea obtenida en cada tratamiento. Para esto, cada muestra fue molida, se pesaron 0,20 $\mathrm{g}$ de material molido y se colocó en un crisol de porcelana $25 \mathrm{ml}$ de capacidad. Se introdujo el crisol con el material molido en un horno de mufla a $500^{\circ} \mathrm{C}$ durante 4 h; cuando bajó la temperatura del horno de mufla se retiró el crisol con las cenizas, se agregaron $20 \mathrm{ml}$ de $\mathrm{HCl} 2 \mathrm{~N}$ y se llevó a ebullición durante 2 min. Se trasvasó el contenido del crisol a una probeta y se llevó a un volumen final de $50 \mathrm{ml}$ con agua destilada; finalmente se trasvasó todo a un tubo de ensayo. En la solución resultante del tubo de ensayo se analizó la concentración de calcio, magnesio, potasio y sodio en $\mathrm{g} \mathrm{kg}^{-1}$, con un espectrofotómetro de absorción atómica (Varian modelo 220 A).

\section{Análisis estadísticos}

En los ensayos con plantas el diseño experimental fue completamente aleatorizado con cinco repeticiones por cada tratamiento.

Tanto a las variables medidas a los sustratos como a los plantines florales, se les realizó análisis de varianza y test de Tukey $(P<0,05)$ para comparación de medias. El software estadístico utilizado fue el programa InfoStat versión 2009 (Di Rienzo et al., 2009).

\section{RESULTADOS Y DISCUSIÓN}

\section{Análisis realizados a los sustratos}

La densidad aparente (Tabla 2) de cada sustrato superó el rango establecido como óptimo, menor a $400 \mathrm{~kg} \mathrm{~m}^{-3}$ (Ansorena Miner, 1994; Abad et al., 2001). Una densidad más baja permitiría un manejo menos dificultoso en cuanto al traslado del sustrato, transporte y relleno de las macetas (Handreck \& Black, 2002; Kämpf, 2005).

El espacio poroso total (Tabla 2) de todos los sustratos superó el valor óptimo de 80\% (Bunt, 1988; Ansorena Miner, 1994; Burés, 1997; Abad et al., 2001, 2004). Hubo diferencias significativas en- 
Tabla 2. Densidad aparente (Dap), espacio poroso total (EPT), porosidad de aireación (PA), capacidad de retención de agua (CRA), pH y conductividad eléctrica (CE) de los sustratos evaluados.

\begin{tabular}{|c|c|c|c|c|c|c|c|c|c|c|c|c|}
\hline \multirow{2}{*}{$\begin{array}{c}\text { Sustratos } \\
\text { CGG } 20 \%+\text { CP } 80 \%\end{array}$} & \multicolumn{2}{|c|}{$\begin{array}{c}\text { Dap } \\
\left(\mathrm{kg} \mathrm{m}^{-3}\right)\end{array}$} & \multicolumn{2}{|c|}{$\begin{array}{l}\text { EPT } \\
(\%)\end{array}$} & \multicolumn{2}{|c|}{$\begin{array}{l}\text { PA } \\
(\%)\end{array}$} & \multicolumn{2}{|c|}{$\begin{array}{c}\text { CRA } \\
(\%) \\
\end{array}$} & \multicolumn{2}{|c|}{$\mathrm{pH}$} & \multicolumn{2}{|c|}{$\begin{array}{c}\mathrm{CE} \\
\left(\mathrm{dS} \mathrm{m}^{-1}\right) \\
\end{array}$} \\
\hline & 590 & $\mathrm{a}$ & 86 & $\mathrm{~cd}$ & 45 & de & 40 & $\mathrm{~cd}$ & 6,3 & g & 0,8 & $f$ \\
\hline CGG $50 \%+$ CP $50 \%$ & 580 & $a b$ & 85 & de & 42 & efg & 43 & $\mathrm{bc}$ & 7,0 & $\mathrm{e}$ & 1,0 & e \\
\hline CGG $80 \%+$ CP $20 \%$ & 550 & bcd & 89 & a & 57 & $a$ & 32 & f & 7,9 & c & 1,1 & d \\
\hline $\mathrm{CGG}_{2} 20 \%+\mathrm{CP} 80 \%$ & 540 & cde & 85 & d & 44 & ef & 41 & bcd & 6,6 & $\mathrm{f}$ & 1,0 & e \\
\hline $\mathrm{CGG}_{2}, 50 \%+\mathrm{CP} 50 \%$ & 530 & de & 84 & ef & 40 & $g$ & 44 & $\mathrm{~b}$ & 7,4 & $d$ & 1,3 & C \\
\hline $\mathrm{CGG}_{2}^{2} 80 \%+\mathrm{CP} 20 \%$ & 560 & bcd & 87 & $b$ & 49 & $\mathrm{~cd}$ & 39 & d & 8,2 & b & 1,6 & b \\
\hline $\mathrm{CGG}_{3}^{2} 20 \%+\mathrm{CP} 80 \%$ & 520 & e & 87 & b & 36 & $\mathrm{~h}$ & 51 & a & 6,6 & f & 1,0 & de \\
\hline $\mathrm{CGG}_{3} 50 \%+\mathrm{CP} 50 \%$ & 540 & de & 83 & $f$ & 40 & $\mathrm{fg}$ & 42 & bc & 7,6 & d & 1,6 & b \\
\hline $\mathrm{CGG}_{3}^{3} 80 \%+\mathrm{CP} 20 \%$ & 570 & $a b c$ & 87 & $b$ & 53 & $\mathrm{~b}$ & 34 & ef & 8,3 & a & 2,1 & a \\
\hline Sustrato comercial & 570 & $a b c$ & 87 & $\mathrm{bc}$ & 51 & $\mathrm{bc}$ & 36 & $\mathrm{e}$ & 5,1 & $\mathrm{~h}$ & 1,1 & de \\
\hline
\end{tabular}

Letras distintas entre filas de una misma columna indican diferencias significativas según el Test de Tukey $(p<=0,05)$. CGG: Compost de guano de gallina CP: Compost de corteza de pino.

tre los sustratos $(p<0,0001)$ : aquellos formulados con $80 \%$ de los tres CGG fueron los de mayor porosidad total, seguidos por los formulados con $20 \%$ de los tres CGG. El sustrato con $80 \%$ de $\mathrm{CGG}_{1}$, superó estadísticamente a los restantes sustratos.

La relación entre los poros con aire y con agua permite valorar la textura y/o estructura del sustrato (Abad et al., 2004). Los sustratos con $20 \%$ y $50 \%$ de los tres compost de guano de gallina tuvieron los mayores porcentajes de la capacidad de retención de agua (Tabla 2); en el sustrato con $20 \%$ de $\mathrm{CGG}_{3}$ se observó el mayor valor $(p<0,0001)$. La porosidad de aireación (Tabla 2), fue mayor en los sustratos formulados con $80 \%$ de los tres CGG, en particular para el sustrato con $80 \%$ de $\mathrm{CGG}_{1}$ que superó significativamente $(\mathrm{p}<0,0001)$ a los demás sustratos.

Estos resultados sugieren que los compost de guano de gallina evaluados aportaron aireación al sustrato y menor capacidad de retención de agua. Sin embargo, considerando los rangos óptimos según Bunt (1988) y Abad et al. (2004) (PA: 20-30\%; CRA: 24-40\%), todos los sustratos presentaron adecuada capacidad de retención de agua y alto porcentaje de poros con aire.

En cuanto a los valores de pH (Tabla 2), los sustratos con $80 \%$ de los tres CGG tuvieron los valores más altos $(7,9-8,3)$, seguidos por aquellos con $50 \%$ $(7,0-7,6)$, superando el rango óptimo establecido para la mayoría de las especies cultivadas en sustratos según Handreck \& Black (2002) (pH entre 5,5 y 6,3 ). Los sustratos con $20 \%$ de los tres CGG y el sustrato comercial se encontraron cercanos o dentro de este rango. El pH tiene gran influencia sobre la disponibilidad de los nutrientes, y algunos de éstos se encuentran más disponibles que otros según su valor (Bunt, 1988; Ansorena Miner, 1994; Burés, 1997). Según estudios realizados por Peterson (1982), en sustratos orgánicos, fosforo, hierro, manganeso, boro, zinc y cobre aumentan su dispo- nibilidad con la disminución del pH, mientras que calcio y magnesio, aumentan su disponibilidad con el incremento del pH. Por lo tanto, el compost de guano de gallina por haber tenido altos valores de $\mathrm{pH}$ (mayor a 8,0), incrementó el pH de los sustratos en forma creciente con el aumento del porcentaje de compost usado en la formulación, aumentando la disponibilidad de calcio y magnesio.

Excepto los sustratos con $50 \%$ y $80 \%$ de $\mathrm{CGG}_{2}$ y $\mathrm{CGG}_{3}$, que presentaron valores de $\mathrm{CE}$ superiores a $1 \mathrm{dS} \mathrm{cm}^{-1}$ (1,3 a 2,1 dS $\mathrm{cm}^{-1}$ ), los restantes sustratos tuvieron valores cercanos al límite (Tabla 2). El sustrato con $80 \%$ de $\mathrm{CGG}_{3}$, se diferenció estadísticamente de todos los sustratos por su mayor valor $(p<0,0001)$. Valores menores a $1 \mathrm{dS} \mathrm{cm}^{-1}$ son recomendables para su uso. Si el sustrato supera este valor, podría haber problemas de salinidad, los cuales dependerán de la edad de la planta, condiciones ambientales, prácticas de manejo y característica de la especie (Abad et al., 2004).

En cuanto al contenido de nutrientes, según los valores de referencia del Instituto de Floricultura del INTA, los niveles de potasio y sodio (Figura 2) fueron aceptables para el sustrato comercial y los sustratos con $20 \%$ de $\mathrm{CGG}_{1}$ y $\mathrm{CGG}_{3}$, pero los restantes sustratos tuvieron niveles altos de ambos elementos. En especial, el sustrato con $80 \%$ de $\mathrm{CGG}_{3}$, el cual se diferenció estadísticamente de los demás sustratos $(p<0,0001)$. Los niveles de potasio en los sustratos con compost de guano de gallina fueron altos en relación a los demás macronutrientes analizados. Las concentraciones de potasio reportados en compost varían entre 0,7 a más de $12 \mathrm{~g} \mathrm{~kg}^{-1}$, con una media de $5,4 \mathrm{~g} \mathrm{~kg}^{-1}$ (Zhenli et al., 2005); los niveles de potasio de los $\mathrm{CGG}_{1}$, $\mathrm{CGG}_{2}$ y $\mathrm{CGG}_{3}$ fueron de $9,37 \mathrm{~g} \mathrm{~kg}^{-1}, 12,43 \mathrm{~g} \mathrm{~kg}^{-1} \mathrm{y}$ $14,68 \mathrm{~g} \mathrm{~kg}^{-1}$ respectivamente, es decir, superiores a los valores medios. Valores similares se encontraron en los compost de residuos de la elaboración cerveza (levadura y malta) + restos de poda de li- 


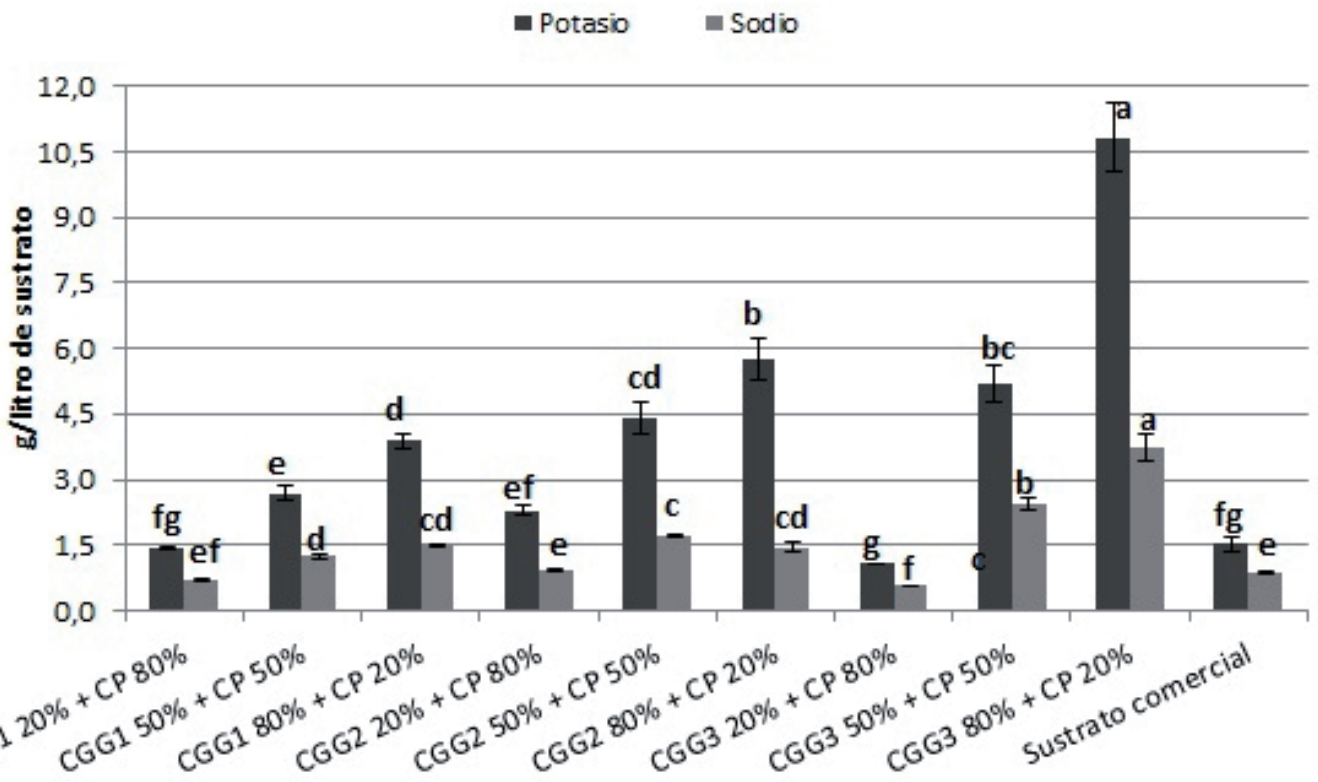

Letras distintas entre barras de un mismo color indican diferencias significativas según el Test de Tukey $(p<=0,05)$. Barras verticales en cada punto indican intervalo de confianza de 95\%. CGG: Compost de guano de gallina CP: Compost de corteza de pino.

Figura 2. Concentración de potasio y sodio de los sustratos evaluados.

- Calcio Magnesio nitratos

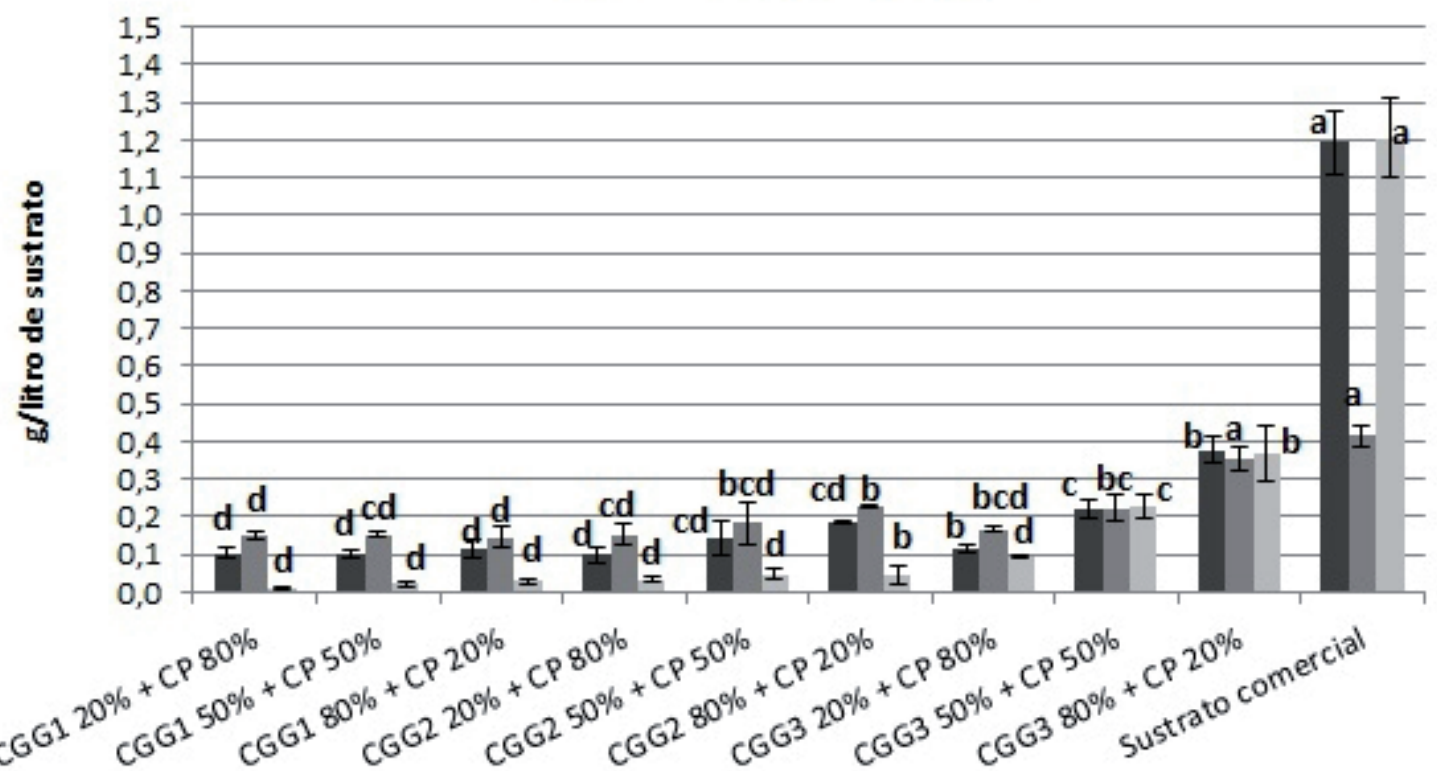

Letras distintas entre barras de un mismo color indican diferencias significativas según el Test de Tukey $(p<=0,05)$. Barras verticales en cada punto indican intervalo de confianza de 95\%. CGG: Compost de guano de gallina CP: Compost de corteza de pino.

Figura 3. Concentración de calcio, magnesio y nitratos de los sustratos evaluados.

moneros, con $8,5 \mathrm{~g} \mathrm{~kg}^{-1}$ de potasio y compost de residuos de oliva + hojas de olivo, con 12,6 $\mathrm{g} \mathrm{kg}^{-1}$ de potasio (Garcia-Gomez et al., 2002).

Los niveles de nitrato, calcio y magnesio (Figura 3) fueron aceptables, según los valores de refe- rencia del Instituto de Floricultura del INTA, para el sustrato comercial y bajos para los demás sustratos. El sustrato comercial se diferenció por la mayor concentración de nitrato y magnesio con los restantes ( $p<0,0001)$. Los sustratos comerciales 


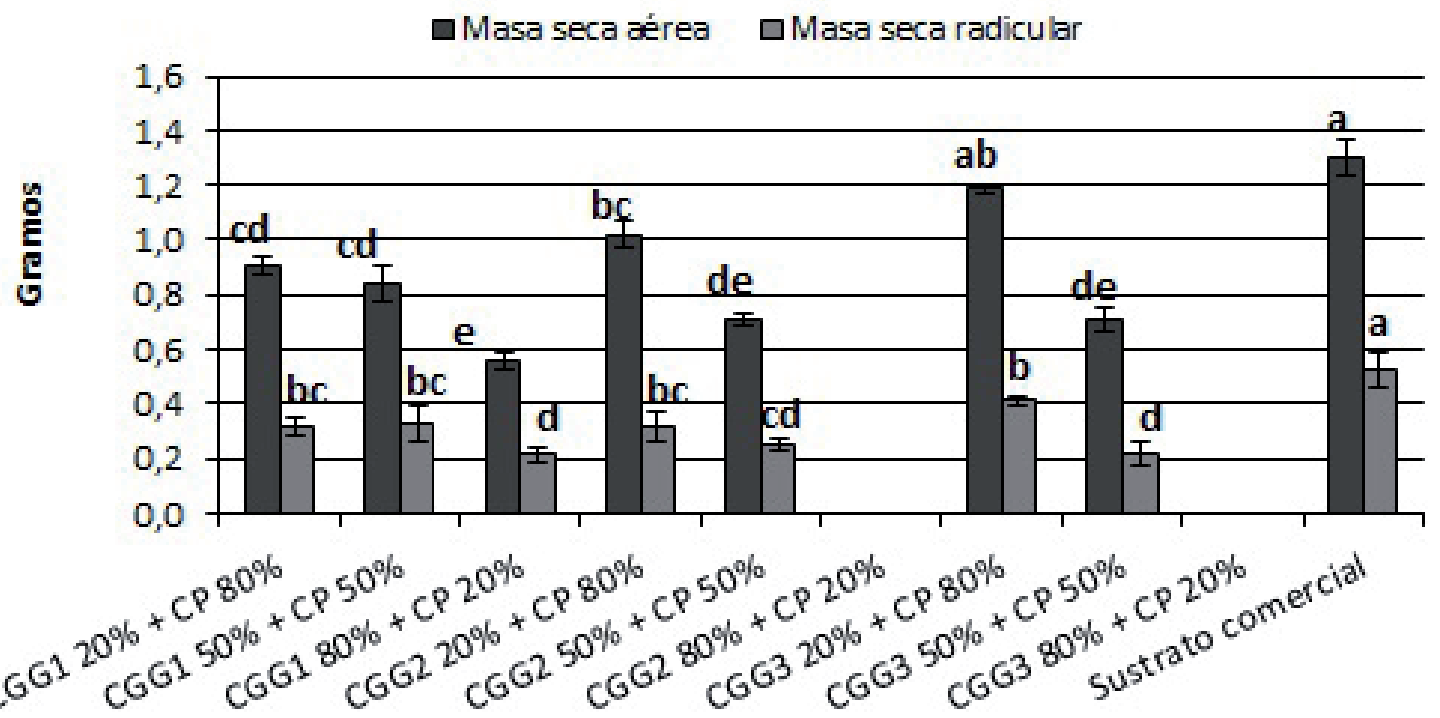

Letras distintas entre barras de un mismo color indican diferencias significativas según el Test de Tukey $(p<=0,05)$. Barras verticales en cada punto indican intervalo de confianza de 95\%. CGG: Compost de guano de gallina CP: Compost de corteza de pino. La ausencia de columnas se debió a la mortandad de las plantas.

Figura 4. Masa seca aérea y radicular de las plantas de coral de cada sustrato evaluado.

tienen una carga de macro y micronutrientes más equilibrada que permiten un crecimiento inicial rápido, y en algunos casos es suficiente para nutrir a la planta en todo el período de almácigo (Favaro et al., 2002); en los siguientes períodos se recomienda fertilizar según los requerimientos de la planta.

\section{Ensayo con plantines florales}

Los plantines de coral (Figura 4) desarrollados en el sustrato comercial lograron la mayor masa seca aérea y radicular (1,30 g y 0,53 g), diferenciándose de los restantes sustratos ( $p<0,0001$ y $p$ $<0,0001$ ), seguido por los plantines desarrollados en el sustrato con $20 \%$ de $\mathrm{CGG}_{1}(0,91 \mathrm{~g}$ y 0,32 g), $\mathrm{CGG}_{2}(1,02 \mathrm{~g}$ y $0,32 \mathrm{~g}), \mathrm{CGG}_{3}(1,19 \mathrm{~g}$ y $0,41 \mathrm{~g})$ y con $50 \%$ de $\mathrm{CGG}_{1}(0,84 \mathrm{~g}$ y 0,32 g).

Resultados similares se obtuvieron en el ensayo con plantines de alegría del hogar (Figura 5): los desarrollados en el sustrato comercial alcanzaron una mayor masa seca aérea y radicular $(0,70 \mathrm{~g}$ y $0,12 \mathrm{~g})$ diferenciándose de los restantes sustratos ( $p<0,0001$ y $p<0,0001$ ), seguido por los plantines desarrollados en el sustrato con 20\% de CGG $(0,42$ g y $0,096 \mathrm{~g}), \mathrm{CGG}_{2}(0,38 \mathrm{~g}$ y $0,079 \mathrm{~g}), \mathrm{CGG}_{3}$ $(0,52 \mathrm{~g}$ y $0,082 \mathrm{~g})$ y con $50 \%$ de $\mathrm{CGG}_{1}(0,37 \mathrm{~g}$ y $0,063 \mathrm{~g})$.

Los sustratos con $50 \%$ de $\mathrm{CGG}_{2}$ y $\mathrm{CGG}_{3}$ y con $80 \%$ de $\mathrm{CGG}_{1}, \mathrm{CGG}_{2}$ y $\mathrm{CGG}_{3}$ tenían CE mayores a
$1 \mathrm{dS} \mathrm{m}^{-1}$. Los plantines de coral desarrollados en los sustratos con $80 \%$ de $\mathrm{CGG}_{2}$ y $\mathrm{CGG}_{3}$, y los plantines de alegría del hogar desarrollados en los sustratos con $80 \%$ de $\mathrm{CGG}_{3}$ murieron a los 3 días después trasplante; los plantines de coral murieron a partir de CE de 1,6 dS m-1, mientras que los de alegría del hogar lo hicieron a partir de 2 dS $\mathrm{m}^{-1}$. Grigatti et al. (2007) utilizaron distintos porcentajes de compost de restos de poda y lodo de depuradora para producir plantines de salvia (Salvia splendens L.), begonia (Begonia semperflorens hybridus), tagete (Tagete erecta L.) y mimulus (Mimulus luteus L.), y también observaron que los plantines de mejor calidad eran los de aquellos sustratos que tenían una CE menor de $1 \mathrm{dS} \mathrm{m}^{-1}$. Esto demuestra la importancia de la CE al formular un sustrato.

En el análisis químico de la masa seca aérea se observó que en los plantines de coral (Tabla 3) y de alegría del hogar (Tabla 4) desarrollados en el sustrato comercial, y en los que contenían $20 \%$ de los tres CGG, se tuvo una mayor concentración de calcio y magnesio y una menor concentración de potasio. Los análisis de la masa seca aérea de los plantines en los sustratos con $80 \%$ y $50 \%$ de los tres CAV dieron mayor contenido de potasio pero menor de calcio y magnesio. Estos resultados sugieren que hubo consumo de lujo de potasio e inhibición de absorción de Ca y Mg (Bunt, 1988). Resultados similares encontraron Bustamante et al. (2008) y Carmona et al. (2012) debido a una mayor 


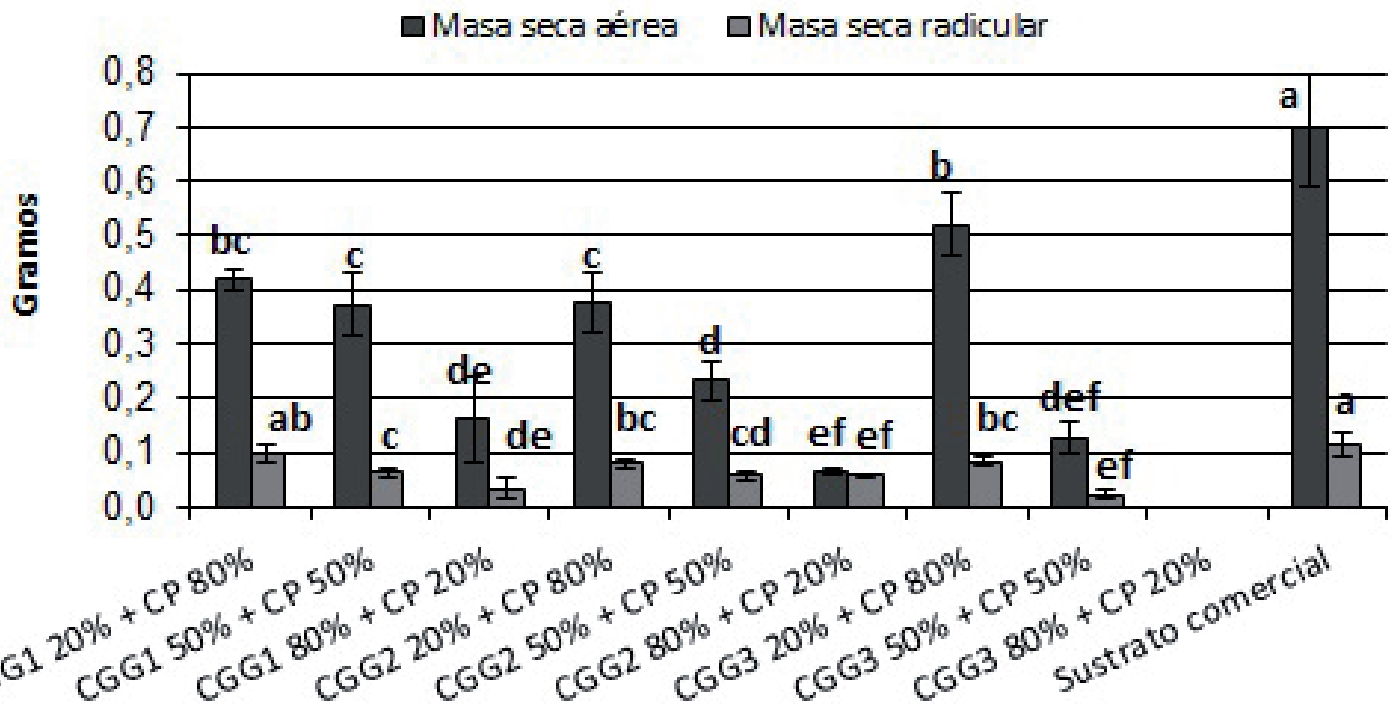

Letras distintas entre barras de un mismo color indican diferencias significativas según el Test de Tukey $(p<=0,05)$. Barras verticales en cada punto indican intervalo de confianza de 95\%. CGG: Compost de guano de gallina CP: Compost de corteza de pino. La ausencia de columnas se debió a la mortandad de las plantas.

Figura 5. Masa seca aérea y radicular de las plantas de alegría del hogar de cada sustrato evaluado.

Tabla 3. Contenido de calcio, magnesio, potasio y sodio de la masa seca aérea de las plantas de coral de cada sustrato evaluado.

\begin{tabular}{|c|c|c|c|c|c|c|c|c|}
\hline \multirow{2}{*}{$\begin{array}{l}\text { Sustratos } \\
\end{array}$} & \multicolumn{2}{|c|}{ Calcio $\left(\mathrm{g} \mathrm{kg}^{-1}\right)$} & \multicolumn{2}{|c|}{ Magnesio $\left(\mathrm{g} \mathrm{kg}^{-1}\right)$} & \multicolumn{2}{|c|}{ Potasio $\left(\mathrm{g} \mathrm{kg}^{-1}\right)$} & \multicolumn{2}{|c|}{ Sodio $\left(\mathrm{g} \mathrm{kg}^{-1}\right)$} \\
\hline & 2,9 & $\mathrm{bc}$ & 2,3 & $\mathrm{bc}$ & 21,7 & $\mathrm{~d}$ & 17,6 & $\mathrm{~cd}$ \\
\hline CGG $50 \%+$ CP $50 \%$ & 2,4 & bcd & 2,0 & $\mathrm{C}$ & 33,1 & bc & 19,3 & bc \\
\hline CGG $80 \%+C P 20 \%$ & 2,0 & $\mathrm{~cd}$ & 2,0 & c & 35,5 & bc & 22,0 & a \\
\hline $\mathrm{CGG}^{1} 20 \%+\mathrm{CP} 80 \%$ & 3,2 & $a b$ & 3,1 & $a b$ & 32,9 & $\mathrm{bc}$ & 20,5 & $a b$ \\
\hline $\mathrm{CGG}^{2} 50 \%+\mathrm{CP} 50 \%$ & 1,7 & d & 2,1 & $\mathrm{C}$ & 39,6 & $a b$ & 22,1 & a \\
\hline $\mathrm{CGG}^{2} 80 \%+\mathrm{CP} 20 \%$ & $\mathrm{SP}$ & & $\mathrm{SP}$ & & $\mathrm{SP}$ & & $\mathrm{SP}$ & \\
\hline $\mathrm{CGG}_{3}^{2} 20 \%+\mathrm{CP} 80 \%$ & 2,6 & bcd & 2,4 & bc & 31,6 & c & 19,1 & bc \\
\hline $\mathrm{CGG}^{3} 50 \%+\mathrm{CP} 50 \%$ & 1,9 & $\mathrm{~cd}$ & 2,2 & $\mathrm{bc}$ & 44,8 & a & 22,4 & a \\
\hline $\mathrm{CGG}_{3}^{3} 80 \%+\mathrm{CP} 20 \%$ & SP & & SP & & SP & & SP & \\
\hline Sustrato comercial & 4,2 & a & 3,5 & a & 23,4 & d & 15,8 & d \\
\hline
\end{tabular}

Letras distintas entre filas de una misma columna indican diferencias significativas según el Test de Tukey $(p<=0,05)$. CGG: Compost de guano de gallina CP: Compost de corteza de pino. SP: Sin plantas por mortandad.

Tabla 4. Contenido de calcio, magnesio, potasio y sodio de la masa seca aérea de las plantas de alegría del hogar de cada sustrato evaluado

\begin{tabular}{|c|c|c|c|c|c|c|c|c|}
\hline \multirow{2}{*}{$\frac{\text { Sustratos }}{\text { CGG }_{1} 20 \%+\text { CP } 80 \%}$} & \multicolumn{2}{|c|}{ Calcio $\left(\mathrm{g} \mathrm{kg}^{-1}\right)$} & \multicolumn{2}{|c|}{ Magnesio $\left(\mathrm{g} \mathrm{kg}^{-1}\right)$} & \multicolumn{2}{|c|}{ Potasio $\left(\mathrm{g} \mathrm{kg}^{-1}\right)$} & \multicolumn{2}{|c|}{ Sodio $\left(\mathrm{g} \mathrm{kg}^{-1}\right)$} \\
\hline & 3,56 & $\mathrm{~b}$ & 3,95 & $\mathrm{~b}$ & 30,18 & $\mathrm{e}$ & 22,18 & $\mathrm{~cd}$ \\
\hline CGG $50 \%+$ CP $50 \%$ & 2,44 & c & 2,91 & c & 49,71 & $\mathrm{~cd}$ & 25,64 & $a b c$ \\
\hline CGG $80 \%+$ CP $20 \%$ & 2,04 & de & 1,94 & de & 67,59 & $a b$ & 29,96 & $a b$ \\
\hline CGG $20 \%+$ CP $80 \%$ & 2,96 & bc & 3,42 & $\mathrm{bc}$ & 43,09 & $d$ & 23,15 & $\mathrm{~cd}$ \\
\hline $\mathrm{CGG}^{2} 50 \%+\mathrm{CP} 50 \%$ & 1,93 & de & 2,08 & de & 59,29 & bc & 25,38 & bc \\
\hline $\mathrm{CGG}_{2}^{2} 80 \%+\mathrm{CP} 20 \%$ & 1,78 & e & 1,86 & $\mathrm{~d}$ & 73,43 & a & 30,37 & a \\
\hline $\mathrm{CGG}_{3}^{2} 20 \%+\mathrm{CP} 80 \%$ & 2,87 & $\mathrm{~cd}$ & 3,43 & bc & 40,78 & d & 23,41 & $\mathrm{~cd}$ \\
\hline $\mathrm{CGG}_{3} 50 \%+\mathrm{CP} 50 \%$ & 2,71 & $\mathrm{c}$ & 2,14 & de & 64,91 & $a b$ & 22,09 & cde \\
\hline $\mathrm{CGG}_{3} 80 \%+\mathrm{CP} 20 \%$ & $\mathrm{SP}$ & & $\mathrm{SP}$ & & SP & & SP & \\
\hline Sustrato comercial & 4,39 & a & 5,6 & $\mathrm{a}$ & 29,70 & $\mathrm{e}$ & 18,78 & de \\
\hline
\end{tabular}

Letras distintas entre filas de una misma columna indican diferencias significativas según el Test de Tukey $(p<=0,05)$. CGG: Compost de guano de gallina CP: Compost de corteza de pino. SP: Sin plantas por mortandad. 
proporción de compost de orujo de uva en el sustrato usado para producir plantines de melón (Cucumis melo L.), lechuga (Lactuca sativa L.), brócoli (Brassica oleracea L.), acelga (Beta vulgaris L.) y cilantro (Coriandrum sativum L.).

Los resultados confirman las conclusiones de Carmona et al. (2012), quienes mencionan a la alta salinidad y a la baja capacidad de retener agua de la mayoría de los compost como unas de sus principales desventajas, y por lo cual es necesario mezclar con otros materiales para formular un sustrato. Los sustratos formulados con menor porcentaje $\left(20 \%\right.$ ) de $\mathrm{CGG}_{1}, \mathrm{CGG}_{2}$ y $\mathrm{CGG}_{3}$, y con $50 \%$ de $\mathrm{CGG}_{1}$, fueron los de mayor capacidad de retención de agua y menor salinidad, y favorecieron el desarrollo de los plantines de coral y alegría del hogar.

El CCG fue elaborado a partir de una mezcla con $40 \%$ de guano de gallina; en cambio, los demás compost contenían un $60 \%$ y este mayor porcentaje incrementó la CE del compost elaborado. Es decir, que la alta salinidad del CAV se podría corregir disminuyendo el porcentaje de guano de gallina en la mezcla inicial, lo que permitiría utilizar el compost elaborado en mayores porcentajes en formulaciones de sustratos.

\section{CONCLUSIONES}

El compost de guano de gallina puro con CE de 1,4 a 2,7 dS $\mathrm{m}^{-1}$ y pH de 8 a 9, puede ser utilizado hasta un $20 \%$ en formulaciones de sustratos compuestos por otros materiales ácidos y de baja salinidad, como el compost de corteza de pino. Si poseen una CE menor a 1,4 y pH menor a 8, podría utilizarse hasta un 50\%, también en formulaciones con materiales ácidos y de baja salinidad.

Si la mezcla formulada para compostar contiene $40 \%$ o menos de guano de gallina, permitiría obtener un compost con menor salinidad.

Con respecto a las propiedades físicas, el compost de guano de gallina aporta aireación al sustrato, por lo tanto, se debería equilibrar con materiales que contribuyan a una mayor capacidad de retención de agua para una mejor relación de poros con agua y aire. Bajo estas condiciones, se podrán obtener plantines de coral y alegría del hogar con características óptimas, similares a las obtenidas en un sustrato comercial.

La viabilidad de utilizar el compost de guano de gallina en mezclas de sustrato para producir plantines florales permite tener otro campo de aplicación para su uso, además de emplearlo en la agricultura como abono y enmienda orgánica de suelos.

\section{BIBLIOGRAFÍA}

Abad, M.; P.F. Martínez, M.D. Martínez y J. Martínez, 1993. Evaluación agronómica de los sustratos de cultivo. Actas de Horticultura 11: 141-154.

Abad, M.; P. Noguera and S. Burés, 2001. National inventory of organic wastes for use as growing media for ornamental potted plant production: case study in Spain. Bioresource Technology 77: 197-200.

Abad, M.; P. Noguera y C. Carrion, 2004. Los sustratos en los cultivos sin suelo. Capítulo 4. En: Urrestarazu Gavilan M. (eds.). Tratado de cultivo sin suelo. Ed. Mundi Prensa. España. pp. 113-158.

Ansorena Miner, J., 1994. Sustratos propiedades y caracterización. Ed. Mundi-Prensa. Madrid. 172 pp.

Barbaro, L. A.; M. A. Karlanian, S. Imhoff y D. E. Morisigue. 2011. Caracterización de la turba subtropical del departamento Islas del Ibicuy (Entre Ríos, Argentina). Agriscientia, 28 (2): 137-145

Barrena Gómez, R.; F. Vázquez Lima and A. Sánchez Ferrer, 2006. The use of respiration indices in the composting process: a review. Waste Manage, 24: 37-47.

Bremner, J.M., 1965. Inorganic forms of nitrogen. Part 2. In: Black, C.A.; E. De Wite; L.E. Ensminger; F.E. Clark (Editors). Methods of soil analysis. American Society of Agronomy, Inc., Madison, USA. pp. 1179-1237.

Bunt, A. C., 1988. Media and mixes for container-grown plants. Ed. Unwin Hyman. London. 309 pp.

Burés, S., 1997. Sustratos. Ed. Agrotecnias. Madrid. 342 pp.

Bustamante, M.A.; C. Paredes, R. Moral, E. Agulló, M.D. Pérez-Murcia and M. Abad, 2008. Composts from distillery wastes as peat substitutes for transplant production. Resour. Conserv. Recycl. 52, 792-799.

Carmona, E.; M.T. Moreno, M. Avilés and J. Ordovás, 2012. Use of grape marc compost as substrate for vegetable seedlings. Scientia Horticulturae 137: 69-74.

De Boodt, M; O. Verdonck and J. Cappaert, 1974. Methods for measuring the waterrelease curve of organic substrates. Acta Horticulturae 37: 2054-2062.

Di Rienzo, J.A.; F. Casanoves, M.G. Balzarini, L. Gonzalez, M. Tablada y C.W. Robledo, 2009. InfoStat versión 2009. Grupo InfoStat, FCA, Universidad Nacional de Córdoba, Argentina. Disponible en <http://www. infostat.com.ar> Consultada el 10/05/2012.

Estévez-Schwarz, I.; S. Seoane, A. Nuñez and M.E. López-Mosquera, 2009. Characterization and Evaluation of Compost Utilized as Ornamental Plant Substrate. Compost Science \& Utilization Vol. 7 (4): 210-219.

Favaro, J.C.; M.A. Buyatti y M.R. Acosta, 2002. Evaluación de sustratos a base de serrín de Salicáceas (Salix sp.) compostados para la producción de plantones. Invest. Agr.: Prod. Prot. Veg. 17 (3): 367-373. 
Fermino, M. H., 2003. Métodos de análisis para caracterización física de sustratos para plantas. Tesis de doctorado. Universidad Federal de Rio Grande Do Sul. Facultad de Agronomia. Puerto Alegre. 250 pp.

Fonteno, W., 1999. Sustratos: Tipos y propiedades físicas y químicas. Capítulo 5. En: REED, D. W. Agua, sustratos y nutrición en los cultivos de flores bajo invernadero. Ed. Ball Publishing E. Unidos y Hortitecnia Ltda. Colombia. pp. 93-124.

Garcia-Gomez A.; M.P. Bernal and A. Roig, 2002. Growth of ornamental plants in two composts prepared from agroindustrial wastes. Bioresource Technology 83: 8183

Grigatti M.; M.E. Giorgioni and C. Ciavatta, 2007. Compost-based growing media: Infuence on growth and nutrient use of bedding plants. Bioresource Technology 98: 3526-3534.

Handreck, K. \& N. Black, 2002. Growing media for ornamental plants and turf. Third edition. A UNSW Press book. Australia. 542 pp.

Hong, C.; Ch. Tongbin, L. Hongtao, G. Ding, Z. Guodi and $Z$. Jun, 2010. The effect of salinity and porosity of sewage sludge compost on the growth of vegetable seedlings. Scientia Horticulturae 124 (3): 381-386.

Iglesias Jiménez, E.; M. T. Barral Silva y F. C. Marhuenda Egea, 2008. Capítulo 11: Indicadores de estabilidad y madurez del compost. En: Moreno Casco, J. \& R. Moral Herrero. Compostaje. Ed. Mundi Prensa. Madrid. 570 pp.

Kämpf, N. A., 2005. Producao comercial de plantas ornamentais. Agro livros. $254 \mathrm{pp}$.

Landis, T.D. and N. Morgan, 2009. Growing media alternatives for forest and native plant nurseries. In: Dumroese, R.K.; L.E. Riley. National Proceedings: Forest and Conservation Nursery Associations. U.S. Department of Agriculture, Forest Service, Rocky Mountain Research Station: 26-31. Disponible en <http:// www.fs.fed.us/rm/pubs/rmrs_p058.html> Consultada el 23/04/2012.

Landis, T. D.; R. W. Tinus, S. E. Mcdonald y J. P. Barnett, 2000. Manual de viveros para Producción de especies forestales en contenedor. Manual agrícola. Departa- mento de Agricultura de los Estados Unidos, Servicio Forestal. $674 \mathrm{pp}$.

Lemaire, F.; A. Dartigues, L. Riviere, S. Charpenteir y P. Morel, 2005. Cultivos en macetas y contenedores: Principios agronómicos y aplicaciones. Ed. MundiPrensa. Madrid. 110 pp.

Ministerio de Agricultura, Ganadería y Pesca de la Nación Argentina, 2010. Anuario ganados y carnes, pp 167-171.

Nelson, E. B.; and H. A. J. Hoitink, 1982. Factors affecting suppression of Rhizoctonia solani en container media. Phytopathology 72: 275-279.

Peterson, J. C., 1982. Effects of pH upon nutrient availability in a commercial soiless roots medium utilized for floral crop production. Ohio State University and Ohio Reseach and Develoment Center. Cir. 268: 16-19.

Raviv, M.; Y. Chen and Y. Inbar, 1986. Peat and peat substitutes as growth media for container-grown plants. In: Chen, Y. and Y. Avnimelech (Eds.), The Role of Organic Matter in Modern Agriculture. Martinus Nijhoff Publishers, Dordrecht, The Netherlands. pp. 257-287.

Raviv, M. and J. H. Lieth, 2008. Soilless culture: theory and practice. Ed. Elseiver. 587 pp.

Spencer, S. and D. M. Benson, 1982. Pine Bark, Hardwood Bark, and Pest Amendment Effect en Developtmen of Phytophthora spp. And Lupine Rood Rot. Phytopathology 72 (3): 347-351.

TMECC (Test methods for the examination of composting and compost), 2001. The US Department of Agriculture and The US Composting Council. Edaphos International, Houston, USA.

Villa Castillo, J., 2004. Inoculating Composted Pine Bark for Container production. Nativeplant Journal 5: 181185.

Zhenli, H.; Xiaoe Yang, A. K. Brian, P. J. Stoffela y D. V. Calvert, 2005. Ventajas de la utilización de compost para la nutrición fosfórica, potásica, cálcica, magnésica y de micronutrientes. Capítulo 15. En: Stoffella, P.J. y B.A. Kahn. Utilización de Compost en los Sistemas de cultivo hortícola. Ed. Mundi Prensa. Madrid. pp. 307-322. 